NOT FOR QUOTATION

WITHOUT THE PERMISSION

OF THE AUTHORS

\title{
A Note On Random Intensities and Conditional Survival Functions
}

Anatoli Yashin

Elja Arjas

December 1986

WP-86-77

Working Papers are interim reports on work of the International Institute for Applied Systems Analysis and have recelved only limited review. Views or opinions expressed herein do not necessarily represent those of the Institute or of its National Member Organizations.

INTERNATIONAL INSTITUTE FOR APPLIED SYSTEMS ANALYSIS A-2361 Laxenburg, Austrla 


\section{Foreword}

One of the interesting directions of research in IIASA's Population Program deals with the methodological aspects of population heterogeneity dynamics. The crucial notion in this analysis is the stochastic intensity which is widely used in the stochastic processes models of human morbidity and mortality or technical failure.

This paper provides the probabilistic specification of this notion which gives an opportunity to use the results of modern general theory of processes in analyzing factors that influence demographic characteristics.

Anatoll Yashin

Deputy Leader

Population Program 


\section{Acknowledgement}

We are grateful to Pentti Laara for a number of good comments. 


\title{
A Note on Random Intensities and Conditional Survival Functions
}

\author{
Anatoli Yashin and Elja Arjas
}

\section{Introduction}

Let $\xi=\left(\xi_{t}\right)_{t \geq 0}$ be a random process and $T$ a random time in some probability space. The intensity, or hazard rate, related to the occurrence of $T$ and given the observation of $\xi_{0}^{t}=\left\{\xi_{s}, 0 \leq s \leq t\right\}$, is often identified as a limit of the form

$$
\lambda(t, \xi)=\lim _{\Delta \downarrow 0} \frac{1}{\Delta} P\left(t<T \leq t+\Delta \mid \xi_{0}^{t} ; T \geq t\right\} .
$$

Does such a definition mean that the corresponding conditional survival function, when $\xi$ is observed, can be obtained from the "exponential formulas"

$$
P\left(T \geq t \mid \xi_{0}^{t}\right)=e^{-\int_{0}^{t} \lambda(s, \xi) d s} \quad ?
$$

Equality (2) is often tacitly assumed in medical and epidemiological studies when dealing with survival analysis in the presence of observable influencing random factors. It turns out that this formula does not always hold.

The exponential formula can be viewed as the solution satisfying $P(T \geq 0)=1$ of a corresponding differential equation. Thus, when there is no conditioning, and assuming absolute continuity of the distribution function $F(t)=P(T \leq t), t \geq 0$, the formulas

$$
\frac{d}{d t} F(t)=-\lambda(t) F(t)
$$

and

$$
F(t)=1-e^{-\int_{0}^{t} \lambda(s) d s}
$$

express a one-to-one correspondence between the hazard rate $\lambda$ and the distribu- 
tion function $F$. This has an obvious extension to the case where conditioning is on a fixed $\sigma$-algebra, say, $G_{0}$ involving the conditional distribution function $F^{C_{0}}(t)=P\left(T \leq t \mid G_{0}\right)$ and the corresponding hazard rate $[1,2,3]$. Why, then, is it that formulas such as (3a) and (3b) do not necessarily hold for meaningful hazard rates when the conditioning is "dynamically" on time dependent random factors?

A first observation is that knowledge of $\xi_{0}^{t}$ may directly tell whether $\{T \leq t\}$ holds or not. In other words, $P\left(T \leq t \mid \xi_{0}^{t}\right)$ may be either 0 or 1 , whereas typical values of the function $1-\exp \left(-\int_{0}^{t} \lambda(s, \xi) d s\right)$ would be strictly between 0 and 1 . As a concrete example, one could think the survival of an individual, assuming that $\xi$ monitors the blood pressure. A second and more formal problem with (2) is that the left-hand side should be defined for all sample points of the probabilistic space while $\lambda(t, \xi)$ in (1) is only partially defined (on $\{T \geq t\}$ ).

In order to settle these questions in the most convenient way we switch over to the currently well-known and extremely flexible formalism involving counting processes and their associated compensators [see e.g. Jacod [2] or Liptcer and Shiryayev [3].

\section{The Results}

Let $N=\left(N_{t}\right)_{t>0}$ with $N_{t}=1_{\{T<t\}}$ be the process which counts "one" at $T$. Let $G=\left(G_{t}\right)_{t 20}$ be the observed history on which the assessment of the T-related hazard is based, and define $H=\left(H_{t}\right)_{t 20}$ by $H_{t}=G_{t} \vee \sigma\left\{N_{s}, s \leq t\right\}$. Clearly, if $T$ is a $G$-stopping time, we have $H=G$. Both $G$ and $H$ are assumed to satisfy "the usual conditions" regarding right-continuity and completeness [4].

It is well known that, under regularity conditions, if $G$ is the $\xi$-generated history, $\lambda(t, \xi)$ of (1) satisfies the requirement

$$
\left(N_{t}-\int_{0}^{t} \lambda(s, \xi) 1_{\mid T \geq s\}} d s\right)_{t \geq 0}=H \text {-martingale } .
$$

The process $\left(\lambda(t, \xi) 1_{\{T \geq t\}}\right)_{t \geq 0}$ is called the stochastic $H$-intensity corresponding to $T$. In fact, (4) is then used directly as the definition of such an intensity, instead of starting from a limit such as (1). 
Let $F=\left(F_{t}\right)_{t \geq 0}$ be the process $F_{t}=P\left(T \leq t \mid G_{t}\right)$. Clearly, $F$ is the ordinary distribution function of $T$ if $G$ is trivial, while $F=N$ if $T$ is a $G$-stopping time. In general $F$ need not be monotone. It is easily verified, however, that $F$ is a $G$ submartingale. We denote the $G$-compensator of $F$ by $A$, i.e., $A=\left(A_{t}\right)_{t \geq 0}$ is the unique increasing $G$-predictable process, with $A(0)=0$, such that the difference $F-A$ is a $G$-martingale (see, e.g. Jacod [2] or Liptcer and Shiryayev [3]). Let $N$ and $H$ be as above, and denote by $\Lambda=\left(\Lambda_{t}\right)_{t \geq 0}$ the $H$-compensator of $N$. Here is the main result of this paper:

Theorem. $\Lambda$ has the representation

$$
\alpha \Lambda_{t}=\frac{d A_{t}}{1-F_{t}-} 1_{\{T \geq t)} .
$$

Proof. First observe that this claim is trivial if $T$ is a $G$-stopping time. In the general case where $G_{t} \subset H_{t}, t \geq 0$, it is enough to prove that (i) $\Lambda$ is $H$-predictable, and (ii) $N-\Lambda$ is an $H$-martingale.

We start with (i). The integrand of $\Lambda_{t}=\int_{0}^{t} \frac{1_{|T \geq s|}}{1-F_{s-}} \alpha A_{s}$ is left-continuous and $H$-adapted, therefore $H$-predictable, while $A$ is $G$-predictable (by definition) and therefore also $\boldsymbol{H}$-predictable. The $\boldsymbol{H}$-predictability of $\Lambda$ follows.

In order to prove (ii), denote first $m=N-\Lambda$. It is clear that $E\left|m_{t}\right|<\infty$ for all $t \geq 0$. Therefore it remains to show that

$$
E\left[m_{t} \mid H_{s}\right]=m_{s} \quad \text { a.s. }
$$

holds for $s<t$. We have

$$
E\left[m_{t} \mid H_{s}\right]=E\left[N_{t} \mid H_{s}\right]-E\left[\Lambda_{t} \mid H_{s}\right] .
$$

The first term on the right-hand side can be written as

$$
E\left[N_{t} \mid H_{s}\right]=N_{S}+1_{\{T>S \mid} \frac{E\left[1_{\{T>s \mid} 1_{\{T \leq t\}} \mid G_{s}\right]}{P\left(T>s \mid G_{S}\right)}
$$

while the second term becomes

$$
\begin{gathered}
E\left[\Lambda_{t} \mid H_{s}\right]=E\left[\int_{0}^{t} 1_{\{T \geq u \mid} \frac{d A_{u}}{1-F_{u-}} \mid H_{s}\right] \\
=\int_{0}^{s} 1_{\{T \geq u \mid} \frac{d A_{u}}{1-F_{u-}}+\frac{1_{\{T>s\}} E\left[\int_{s}^{t} 1_{\{T \geq u \mid} \frac{d A_{u}}{1-F_{u-}} \mid G_{s}\right]}{P\left(T>s \mid G_{s}\right)} .
\end{gathered}
$$


Therefore, (6) is equal to

$$
\left.E\left[m_{t} \mid H_{s}\right]=m_{s}+\frac{1_{\{T>s \mid}}{P\left(T>s \mid G_{s}\right)}\left\langle E\left[1_{\{T>s\}}\right\}_{\{T s t\}}\right| G_{s}\right]-E\left[\int_{s}^{t} 1_{\{T \geq u \mid} \frac{d A_{u}}{1-F_{u-}} \mid G_{s}\right] .
$$

However, here the second term vanishes, because, by the well-known properties of the compensator.

$$
\begin{gathered}
E\left[\int_{s}^{t} 1_{\{T \geq u \mid} \frac{d A_{u}}{1-F_{u-}} \mid G_{s}\right]=E\left[\int_{s}^{t}\left(1-N_{u-}\right) \frac{d A_{u}}{1-F_{u-}} \mid G_{s}\right] \\
=E\left[\int_{s}^{t} d A_{u} \mid G_{s}\right]=E\left[1_{\{T>s\}} 1_{\{T \leq t\}} \mid G_{s}\right] .
\end{gathered}
$$

We now show how this theorem can be used in our problem concerning the exponential formula. For this we need the following two conditions:

(C1): $F=\left(F_{t}\right)_{t \geq 0}$ is absolutely continuous ;

(C2): $F$ is of finite variation .

Under these conditions we have $A=F$, the theorem implies in an obvious way the solution to our problem. We have, when denoting $d \Lambda_{t}=\lambda_{t} d t$, the following result.

Carollary. Suppose (C1) and (C2). Then, denoting

$$
Y_{t}=\frac{\frac{d}{d t} F_{t}}{1-F_{t}}, t \geq 0
$$

the stochastic $B$-intensity corresponding to $T$ is given by $\lambda_{t}=Y_{t} 1_{\{T \geq t\}}, t \geq 0$.

Although the proof is obvious from the Theorem, some comments on this result should be helpful. Firstly, (7) is clearly equivalent to

$$
P\left(T \leq t \mid G_{t}\right)=1-e^{-\int_{0}^{t} Y_{2} d s}, t \geq 0 .
$$

(assuming that $\left.P\left(T>0 \mid G_{0}\right)=1\right)$. The crucial point here is not the equivalence of (7) and (8), but the fact that $Y=\left(Y_{t}\right)_{t \geq 0}$, being multiplied by $1_{\{T \geqslant t\}}$, is the $H$ intensity for $T$. 
Secondly, (C1) is clearly necessary for (7) to be a meaningful definition, and for (8) to hold. However, (C2) may need a comment. Here is a simple sufficient condition for (C2):

$\left(C 2^{\prime}\right)$ : For all $t \geq 0, P\left(T \leq t \mid G_{t}\right)=P\left(T \leq t \mid G_{\infty}\right)$ a.s.

The reason is that under (C2') $F$ becomes monotone. (C2') postulates the conditional independence between $\{T \leq t\}$ and $G_{\infty}$ given by $G_{t}$. Using the terminology of Pitman and Speed (1973), one can say that $T$ satisfying (C2') is a randomized $G$-stopping time.

Notice that our conditions for ( 8 ) are actually quite subtle: If $T$ is a $G$-stopping time, (C2') is clearly met; however, (C1) cannot then hold. In a sense, therefore, we must think of $G$, or of $\xi$, as information exogenous to the actual counting process $N$.

\section{Conclusion}

Mathematical models based on counting processes and martingales have proved extremely useful in many applied fields, such as biostatistics, reliability theory, and risk analysis. The most important asset of this approach is its flexibility combined with the powerful methods of the stochastic calculus. As this study shows, however, one should be very cautious when assuming that well-known formulas, such as the exponential formula here, automatically have formally similar extensions.

Lastly, a word about extending our results to more general point processes. Above, we only considered "the single point process" $N_{t}=1_{\{T \leq t\}}, t \geq 0$. If there are more points, say at $(0<) T_{1}<T_{2}<\cdots$, we could easily switch into the counting process $N_{t}=\sum_{i \geq 1} 1_{\left\{T_{i} \leq t\right\}}, t \geq 0$. Therefore $N$ is the sum of "single point processes", and the corresponding $\boldsymbol{H}$-compensator is automatically a sum of processes like (5), each corresponding to some particular point $T_{i}$. A similar extension of the Theorem holds for marked point processes.

On the other hand, the Corollary does not seem to generalize in a useful manner. The formal generalization of the exponential formula would be

$$
\prod_{i}\left(1-F_{t}(i)\right)=e^{-\sum_{i} \int_{0}^{t} Y_{s}(i) d s}, t \geq 0 ;
$$

however, the left-hand side does not appear to have interesting interpretations. 


\section{REFERENCES}

[1] Chou, C.S. and P.A. Meyer (1974) Sur la Representation des Martingales comme Integrales Stochastiques dans les Processus Ponctuels. Lecture Notes in Mathematics, 381 . Berlin: Springer-Verlag.

[2] Jacod, J. (1975) Multivariate Point Processes: Predictable Projection, radonNicodim Derivatives, Representation of Martingales. Zeitschrift für Wahrscheinlichkeitstheorie und Verwandte Gebieten 31:235-253.

[3] Liptcer, R.S. and A.N. Shiryayev (1978) Statistics of Random Processes, Vol. II, Applications. Heidelberg: Springer-Verlag.

[4] Dellacherie, C. and P.A. Meyer (1982) Probabilities and Potential. North Holland.

[5] Pitman, J.W. and T.P. Speed (1973) A Note on Random Times. Stochastic Processes Appl. 1:369-374. 